\title{
Does energy consumption endanger public longevity? An analysis of 34 major energy-consuming countries
}

\author{
Tiansen Liu ${ }^{\mathrm{a}}$, Dapeng Liang ${ }^{\mathrm{a}}$, Xinpeng Xing ${ }^{\mathrm{b}}$, Pei Kuang ${ }^{\mathrm{c}}$, Yufeng Zhang ${ }^{\mathrm{c}}$ \\ ${ }^{a}$ School of Management, Harbin Institute of Technology, 92 West Dazhi Street, Harbin, 150001, China \\ ${ }^{b}$ School of Business, Jiangnan University, 1800 Lihu Avenue, Wuxi, 214122, China \\ ${ }^{c}$ Business School, University of Birmingham, Edgbaston Park Road, Birmingham, B15 2TY, the United Kingdom
}

\begin{abstract}
Energy consumption mode largely affects the quality of public living environment. We examine the effect of energy consumption on public longevity based on the data of 34 major energy-consumption countries. Our results show that both energy use per capita (use scale) and GDP per unit of energy use (use efficiency) push forward the rise of public longevity with the former effect more positive. This trend is also applicable to energy exporting and importing countries as well as high and middle-income countries of our sample. Further, GHG emission per capita and PM 10 concentration mainly caused by energy consumption weakly endanger public longevity. In addition, both education and healthcare resources help to enhance public longevity. Heterogeneity test shows that public longevity in energy importing and high-income countries is more sensitive to the change of pollutants emission than that in energy exporting and middle-income countries. Briefly, energy consumption does not severely endanger public longevity, hence compared with declining consumption scale, reducing pollutants emission should be first addressed.
\end{abstract}

Keywords: Energy consumption, pollutants emission, healthcare resources, public longevity, major energyconsuming countries

\section{Introduction}

The harm of air pollution on public health was empirically supported, while it is unclear whether this harm should be entirely attributed to energy consumption, and such gap will affect the future trend of energy consumption [1]. The US government's withdrawal from the Paris Agreement also implies that the negative effect of energy consumption is not quite severe in some countries. Once the consumption scale is substantially declined, the potential loss may be irreversible. Prior studies implied that there is no need to substantially decline energy consumption scale in major energy-consuming countries [2-3], while some others argued that uncontrolled fossil energy consumption is originally threatening public health, and environmental crisis caused by energy-intensive sectors should be addressed by declining the energy consumption scale or adjusting consumption structure [4-5]. The rise of both total fossil energy consumption and public longevity enlightens us to examine their causality. In this study, we examine the effect of energy consumption and pollutants emission on public longevity to show which link more negatively affects public health based on the data of 34 major energy-consuming countries.

\section{Research Design}

\subsection{Data collection}

Except $\mathrm{PM}_{10}$ concentration collected from the Economy Prediction System Global Statistics Database, all other variables are from the World Bank. Given the close relationship between energy consumption and GDP as well as the integrity of data, we select 34 countries (US, China, Japan, Germany, UK, Italy,

\footnotetext{
* Manuscript received December 20, 2017; revised May 27, 2019.

Corresponding author. Tel.: +13845105537; E-mail address: tiansen0328@hotmail.com.

doi: $10.12720 /$ sgce.8.5.562-567
} 
Canada, South Korea, Russia, Australia, Spain, Mexico, Netherlands, Turkey, Saudi Arabia, Argentina, Poland, Belgium, Thailand, Iran, Austria, Egypt, South Africa, Denmark, Malaysia, Singapore, Columbia, Ireland, Chile, Portugal, Greece, Peru, Czech, and Kazakhstan) whose average proportion of fossil energy consumption was higher than $70 \%$ during 1995 to 2015 with GDP (current US\$) ranking top 50 in 2015 as the sample. With regard to the sample selection, we add following remarks. First, as Venezuela's GDP that is always within top 50 has not published by the World Bank in 2015, it is not selected as the sample. Second, the United Arab Emirates, Hong Kong, and Israel are also not selected given the data missing of some indicators. As there is a small amount of data not being updated to 2014 and 2015, we estimate them based on the changing trend of prior data.

\subsection{Variables and model design}

Dependent variable: We select Life expectancy at birth (year, $L E$ ) as dependent variable to reflect the level of public health. Overall, the $L E$ of our sample keeps a rising trend except South Africa.

Independent variable: Given the close relationship among energy consumption, social progress, and demographic factors, we first select Energy use per capita ( $k g$ of oil equivalent, EUPC) and GDP per unit of energy use (constant 2011 PPP \$ per kg of oil equivalent, GDPEU) as independent variables [6]. Further, given various forms of pollutants emission caused by energy consumption, we select Greenhouse gas emission per capita (metric ton of $\mathrm{CO}_{2}$ equivalent, $\mathrm{GHG}$ ) and $\mathrm{PM}_{10}$ concentration (microgram per cubic meter, $P M_{10}$ ) as another group of independent variable. We separately examine the effect of two groups of independent variables on $L E$ to avoid the multicollinearity.

Control variable: We do not select variables related to public living quality (e.g., GDP per capita) and industrial scale (e.g., industry value added) as control variables because their effects on $L E$ may already cover the effect of energy consumption. Specifically, we design following control variables. (1) The demographic factor. As urban scale and population concentration usually positively affect energy demand, and climate improvement is better for urban residents' health than rural residents, we first select Population density (people per sq. $\mathrm{km}$ of land area, $P D$ ) and Population in urban agglomeration of more than 1 million (\% of total population, $P A$ ) as control variables [6-7]. (2) Social resources allocation. Given the positive effect of education and employment opportunities on public health, we select Unemployment (\% of total labor force, UEM) and Education expenditure (\% of GNI, EE) as control variables [8-9]. (3) Healthcare resources. We finally select Improved sanitation facilities (\% of population with access, ISF), Health expenditure (\% of GDP, HEPC), and Public health expenditure (\% of GDP, $H E P$ ) as control variables because no matter what causes regional or seasonal diseases, healthcare system is always a basic for improving public health [2].

We use the Fixed Effect Model to develop the empirical test. By examining the whole trend of our sample, we find that some factors will not change over time but affect the level of other variables, namely, playing the fixed effect. Further, empirical results are more applicable to show the relationship among variables within our sample rather than the whole world. In addition, the Hausman test shows that the values of Prob $>F$ in each designed model are all less than 0.01 , which also supports the rationality of Fixed Effect Model. Accordingly, we design following measuring models.

$$
\begin{aligned}
& Y_{i t}=\alpha+\beta * C_{i t}+\gamma * X_{1 i t}+\text { country }_{i}+\text { year }_{t}+\varepsilon_{i t} \\
& Y_{i t}=\alpha+\beta * C_{i t}+\gamma * X_{2 i t}+\text { country }_{i}+\text { year }_{t}+\varepsilon_{i t}
\end{aligned}
$$

where $Y_{i t}$ is dependent variable, and $C_{i t}$ is control variable. $X_{1 i t}$ and $X_{2 i t}$ are independent variables that correspond to energy consumption and pollutants emission, respectively. country $y_{i}$ is the country fixed effect that eliminates the indicator not remarkably changing over time (e.g., climatic factors). year ${ }_{t}$ is the time fixed effect that eliminates macroeconomic factors affecting the level of national economy and 
social development in a given year (e.g., political system). $\beta$ is the corresponding coefficient of control variables to dependent variable, and $\gamma$ is the corresponding coefficient of independent variables to dependent variable. Finally, $\alpha$ is the constant term, and $\varepsilon_{i t}$ is the random error term.

\section{Regression Analysis and Robustness Test}

Sensitivity analysis shows that dependent variable is sensitive to the change of explanatory variables. We further conduct the robustness test to show whether regression results will vary with the change of time parameters after regression test by designing the interaction term between each variable and time $(t)$. Table 1 shows both results of fixed-effects regression and robustness test.

Table 1. Results of fixed-effects regression (Model 1 and Model 2) and robustness test (Model 3 and Model 4)

\begin{tabular}{|c|c|c|c|c|c|}
\hline Variable & Model 1 & Model 2 & Variable & Model 3 & Model 4 \\
\hline Constant & $47.3886^{* * * *}(4.9272)$ & $54.3416^{* * * *}(7.7494)$ & Constant & $48.0181^{* * *}(4.6549)$ & $52.8609^{* * * *}(7.4167)$ \\
\hline$P D$ & $0.0003(0.0003)$ & $0.0017^{* * * *}(0.0003)$ & $P D \times t$ & $1.82 \mathrm{e}-07(1.44 \mathrm{e}-07)$ & $8.33 \mathrm{e}-07^{* * *}(1.28 \mathrm{e}-07)$ \\
\hline$P A$ & $-0.0550^{*}(0.1120)$ & $0.0744^{* *}(0.1240)$ & $P A \times t$ & $-3.08 \times 10^{-5^{*}}\left(5.52 \times 10^{-5}\right)$ & $2.88 \times 10^{-5}(0.0001)$ \\
\hline$U E M$ & $0.0282^{*}(0.0393)$ & $-0.0885^{* * *}(0.0521)$ & $U E M \times t$ & $1.35 \times 10^{-5^{*}}\left(1.89 \times 10^{-5}\right)$ & $-4.16 \times 10^{-5 * *}\left(2.58 \times 10^{-5}\right)$ \\
\hline$E E$ & $0.2194^{* * *}(0.2302)$ & $0.2200^{* *}(0.2483)$ & $E E \times t$ & $0.0001^{* * *}(0.0001)$ & $0.0001^{* * * *}(0.0001)$ \\
\hline$I S F$ & $0.1372^{* * * *}(0.0553)$ & $0.1738^{* * * *}(0.0745)$ & $I S F \times t$ & $0.0001^{* * *}\left(2.63 \times 10^{-5}\right)$ & $0.0001^{* * * *}\left(3.48 \times 10^{-5}\right)$ \\
\hline$H E P C$ & $0.1575^{* * *}(0.0924)$ & $0.2617^{* * *}(0.1228)$ & $H E P C \times t$ & $0.0001^{* * *}\left(4.35 \times 10^{-5}\right)$ & $0.0001^{* * *}(0.0001)$ \\
\hline$H E P$ & $0.6552^{* * *}(0.1944)$ & $0.6906^{* * *}(0.2400)$ & $H E P \times t$ & $0.0003^{* * *}(0.0001)$ & $0.0003^{* * *}(0.0001)$ \\
\hline$E U P C$ & $1.5738^{* * *}(0.2921)$ & & $E U P C \times t$ & $0.0008^{* * *}(0.0001)$ & \\
\hline$G D P E U$ & $0.7218^{* * *}(0.0989)$ & & $G D P E U \times t$ & $0.0003^{* * *}\left(4.72 \times 10^{-5}\right)$ & \\
\hline$G H G$ & & $-0.0280(0.0326)$ & $G H G \times t$ & & $-1.09 \times 10^{-5}\left(1.57 \times 10^{-5}\right)$ \\
\hline$P M_{10}$ & & $-0.0542^{* * *}(0.0273)$ & $P M_{10} \times t$ & & $-2.31 \times 10^{-5 * * *}\left(1.32 \times 10^{-5}\right)$ \\
\hline F-value & $206.41^{* * *}$ & $126.52^{* * *}$ & F-value & $217.02^{* * *}$ & $134.24^{* * *}$ \\
\hline Adjusted $\mathrm{R}^{2}$ & 0.73 & 0.63 & Adjusted $\mathrm{R}^{2}$ & 0.74 & 0.64 \\
\hline
\end{tabular}

Table 1 shows that the effect of explanatory variables on $L E$ is robust. Further, we deeply discuss empirical results. First, GDPEU positively affects $L E$, and the positive effect of $E U P C$ is stronger. In contrast, the negative effect of $P M_{10}$ is significant while $G H G$ not. Based on the measurement unit of independent variables, energy consumption greatly contributes to the rise of public longevity. The rise of one unit of EUPC and GDPEU will push forward $L E 1.5738$ and 0.7218 years, respectively, while one unit of $P M_{10}$ will only cause the fall of $L E 0.0542$ years, which implies that the harm of pollutants emission can be well controlled. Overall, although $L E$ is wholly fluctuating, the positive effect of energy consumption keeps strong while pollutants emission opposite.

Results further support the positive effect of energy consumption that is improving public living quality and social welfare largely relies on such consumption. Thus although natural disasters, extreme weather, and heavy pollution caused by energy consumption can endanger public health within some areas, we cannot deny its positive effect within the whole country, and GDPEU is generally showing a sustained rising trend. Further, based on the World Bank's explanation, enhancing GDPEU will help to decline pollutants emission scale, thereby improving the level of public health, while a critical premise of this state is the fall or steady of total energy consumption. Except Japan, Germany, UK, and Belgium, all other countries' total energy consumption is rising, which may weaken the positive effect of GDPEC in the long-term. However, the positive effect of $E U P C$ on $L E$ does not mean that energy consumption should be expanded, while it supports that the positive effect of energy consumption currently outweighs the negative effect. In the case of the rising trend of GDPEC and population scale, it needs to control the total consumption to avoid the reversal of this positive effect. From the macro perspective, even if it appears events (e.g., the seasonal haze) that endanger public health, they will be rapidly addressed by government. Thus even if EUPC and major pollutants emission will show a rising trend, they may not cause the fall of $L E$. 
Second, based on regression results and variables' measurement unit, healthcare resources strongly affect $L E$ even with a decisive role. Here, we describe the effect of healthcare resources as follows. Although new energy alternative and low-carbon technology are widely accepted around the world, there are always some countries that do not have the capability to conduct such programs. To achieve social progress, they will still rely on fossil energy in the coming period, hence GDPEU may not be rapidly enhanced, and pollutants emission may also keep rising. However, public concern on the healthcare can help to weaken such adverse trend.

Third, education investment positively affects $L E$. The positive effect of education is greater than public general cognition because education starts at the young stage, and it determines the formation of social cognition, code of conduct, and mental health [10]. Education usually positively affects the employment rate and residents' income, and with the improvement of healthcare system, residents with higher education level tend to pursue healthier lifestyle. The rising education investment means that our sample pays a high attention to education career. Education and healthcare resources jointly constitute the primary driving force of keeping public health, hence the negative effect of energy consumption and pollutants emission will be weakened by better social service resources.

\section{Heterogeneity Test}

\subsection{Energy exporting and importing countries}

Our sample includes 13 energy exporting and 21 importing countries. Heterogeneity test of this group eliminates the effect of residents' income, thereby showing the role of energy endowment. We only show the effect of independent variables on $L E$ (Table 2) given the limited space.

Table 2. The heterogeneity test of energy exporting (Model 1 and Model 2) and importing countries (Model 3 and Model 4)

\begin{tabular}{lllll}
\hline Variable & Model 1 & Model 2 & Model 3 & Model 4 \\
\hline EUPC & $0.9302^{* * *}(0.3029)$ & & $1.7678^{* * * *}(0.4160)$ & \\
GDPEU & $0.6117^{* * *}(0.1545)$ & & $0.6915^{* * *}(0.1325)$ & $-0.1670^{* * * *}(0.1250)$ \\
$G H G$ & & $-0.0133(0.0156)$ & & $-0.1144^{* * * *}(0.0427)$ \\
$P M_{10}$ & & $-0.0372^{* * *}(0.0217)$ & & $222.66^{* * * *}$ \\
F-value & $37.38^{* * *}$ & $31.69^{* * *}$ & $346.24^{* * *}$ & 0.83 \\
Adjusted R & 0.57 & 0.53 & 0.88 & \\
\hline
\end{tabular}

Table 2 shows that both effects of energy exporting and importing countries' EUPC and GDPEU on $L E$ are significantly positive, also similar with Table 1 , which further supports the positive value of enhancing energy use efficiency. However, although pollutants emission in both kinds of countries negatively affects $L E$, only the coefficient in importing countries is significant. The average $G H G, P M_{10}$, and $L E$ in importing countries are $13.76,63.52$, and 72.14 , respectively, while exporting countries are $9.67,40.57$, and 77.60 with following potential reasons. First, as the advantage of energy endowment in exporting countries, their control on energy consumption scale and use efficiency may be weaker than importing countries, hence it will cause higher EUPC, lower GDPEU, and larger-scale pollutants emission. In contrast, $L E$ in importing countries is more sensitive to the change of pollutants emission probably because more emissions also enhance energy import costs, and the uncertainty of such cost conversely pushes forward to more efficient behaviors on energy consumption. In addition, the average $L E$ in exporting countries is far lower than that in importing countries because old people are more sensitive to environmental quality [11], hence a slight rise of negative environmental factors may cause major health harms in importing countries. Overall, there is a slight heterogeneity between energy exporting and importing countries on effects of energy consumption and pollutants emission.

\subsection{High and middle-income countries}

Our sample includes 21 high-income and 13 middle-income countries. Heterogeneity test of this group eliminates the effect of energy endowment, thereby showing the role of residents' income. Table 3 shows the effect of independent variables on $L E$. 
Table 3. The heterogeneity test of high (Model 1 and Model 2) and middle-income countries (Model 3 and Model 4 )

\begin{tabular}{lllll}
\hline Variable & Model 1 & Model 2 & Model 3 & Model 4 \\
\hline EUPC & $1.4796^{* * * *}(0.4193)$ & & $1.4367^{* * * *}(0.3484)$ & \\
$G D P E U$ & $0.7351^{* * * *}(0.1532)$ & & $0.4357^{* * *}(0.2092)$ & \\
$G H G$ & & $-0.0484^{* * * *}(0.0399)$ & & $0.1564^{* * *}(0.0742)$ \\
$P M_{10}$ & & $-0.0638^{* * * *}(0.0356)$ & & $-0.0154(0.0354)$ \\
F-value & $314.60^{* * *}$ & $157.76^{* * *}$ & $45.53^{* * *}$ & $40.74^{* * *}$ \\
Adjusted $\mathrm{R}^{2}$ & 0.87 & 0.78 & 0.62 & 0.59 \\
\hline
\end{tabular}

Table 3 shows that both effects of high and middle-income countries' EUPC and GDPEU on LE are similar with Table 1 . As the large difference of average $L E$ between high (78.65) and middle-income countries (70.44), energy consumption scale and use efficiency in middle-income countries will be more positively related to social progress as a whole. As the average consumption scale (1.92) and use efficiency (8.57) in middle-income countries are both lower than that in high-income countries (4.15 and 9.22), we infer that consumption scale and use efficiency will rise in middle-income countries, and government may keep investing in such consumption mode. With regard to the effect of $G H G$ and $P M_{10}$, they both significantly negatively affect $L E$ in high-income countries, while the effect of $G H G$ is positive in middle-income countries. The first potential reason is that the average $P M_{10}$ in middle-income countries (69.94) is almost twice as high-income countries (36.60), while the level of GHG (7.83) is lower than high-income countries (13.34). Second, $L E$ in middle-income countries is lower, while as healthcare resources positively affect public health, there will be a great rising potential in $L E$. Overall, the effect of pollutants emission shows the heterogeneity between high and middle-income countries.

\section{Conclusion, Limitation, and Future Research}

We examine the effect of energy consumption on public longevity within 34 major energy-consuming countries. Results show that both energy use per capita and GDP per unit of energy use significantly positively affect public longevity, with former effect more positive. In contrast, the effect of pollutants emission on public longevity is slightly negative. In addition, both education and healthcare resources significantly positively affect public longevity. Further, robustness test shows that with the change of time, the effect of explanatory variables shows a steady trend. Heterogeneity test shows that the effects of energy consumption in energy exporting and importing countries as well as high and middle-income countries are both similar with the whole trend. However, the public longevity in energy importing and high-income countries is more sensitive to the change of pollutants emission than that in energy exporting and middle-income countries, which means that the effect of pollutants emission on public longevity depends on national energy endowment and residents' income. Briefly, energy consumption does not severely endanger public longevity within our sample. As fossil energy is hard to be rapidly replaced, major energy-consuming countries should more focus on reducing cleaner production.

This study is not without limitations. First, although we use public longevity to reflect the level of public health, health problems caused by energy consumption are still concentrated on the incidence of certain diseases that mainly appear in manufacturing centralized, densely populated, and wealthy areas. However, we have not extracted typical energy consumption areas in countries, which may make our results hard to completely show the social effect of energy consumption in high-energy demand areas. Second, this study shows the whole trend in major energy-consuming countries, while the state of each sample may differ from it, hence a comparative study among countries need to be further organized. Third, we select variables as fully as possible, while there are still some variables not being selected given the data missing (e.g., poverty headcount ratio). Fourth, we examine the instantaneous effect of explanatory variables, while the potential lag effect of some variables may affect the change of $L E$. Thus there may be a slight difference between the coefficients of some variables and their real effect.

We suggest focusing on following aspects in future research. First, enlightened by prior studies that separately analyzed the input-output process of energy consumption at national and household levels, input-output functions related to energy trade, sector economic growth, and residents' income should be designed to identify more participating factors that are not easily observed and visually show the behavior 
pattern of various participants [12]. Second, it needs to analyze how public health conversely affects future energy consumption as well, and such feedback mechanism will help to explain how national energy system will be optimized in the future. In other words, the relationship between energy consumption and public health should be further involved in the bi-directional causality. Third, the comparative study among countries usually lacks the numerical simulation for the effect of influential policies, hence it needs to analyze how the effect of important variables will evolve within specific energy policy scenarios. This step will provide robust evidence for optimizing controversial policies.

\section{Acknowledgements}

We thank the funding support from the Natural Science Foundation of China (No. 71774039).

\section{References}

[1] Pope III CA, Ezzati M, Dockery DW. Fine-particulate air pollution and life expectancy in the United States. The New England Journal of Medicine, 2009; 360(4):376-386.

[2] St. Louis ME, Hess JJ. Climate change impacts on and implications for global health. American Journal of Preventive Medicine, 2008; 35(5):527-538.

[3] Jackson SC. Parallel pursuit of near-term and long-term climate mitigation. Science, 2009; 326(5952):526-527.

[4] McMichael AJ, Powles JW, Butler D, Uauy R. Food, livestock production, energy, climate change, and health. Lancet, 2007; 370(9594):1253-1263.

[5] Holdren JP. Science and technology for sustainable well-being. Science, 2008; 319(5862):424-434.

[6] Milner J, Davies M, Wilkinson P. Urban energy, carbon management (low carbon cities) and co-benefits for human health. Current Opinion in Environmental Sustainability, 2012; 4(4):398-404.

[7] Kammen DM, Sunter DA. City-integrated renewable energy for urban sustainability. Science, 2016; 352(6288): $922-928$.

[8] Crimmins EM, Saito Y. Trends in healthy life expectancy in the United States, 1970-1990: gender, racial, and educational differences. Social Science \& Medicine, 2001; 52(11):1629-1641.

[9] Kennelly B, O’Shea E, Garvey E. Social capital, life expectancy and mortality: a cross-national examination. Social Science \& Medicine, 2003; 56(12):2367-2377.

[10] McGill N. Social determinants of health: education attainment linked to health throughout life span. American Journal of Public Health, 2016; 106(10):1719.

[11] Roukos DH. Longevity with systems medicine? epigenome, genome and environment interactions network. Epigenomics, 2012; 4(2):119-123.

[12] Kok R, Benders RMJ, Moll HC. Measuring the environmental load of household consumption using some methods based on input-output energy analysis: a comparison of methods and a discussion of results. Energy Policy, 2006; 34(17):2744-2761. 